\title{
Effect of the treatment of long-range forces on the dynamics of ions in aqueous solutions
}

\author{
Lalith Perera, Ulrich Essmann, and Max L. Berkowitz \\ Department of Chemistry, University of North Carolina, Chapel Hill, North Carolina 27599
}

(Received 25 July 1994; accepted 15 September 1994)

\begin{abstract}
The goal of the present work is to study the dependence of the limiting ionic mobility of such anions as fluoride, chloride, and bromide in water on the way the long-range forces are treated in the computer simulations. With this in mind we have performed molecular dynamics computer simulations where the long-range electrostatic forces were treated using: (a) simple truncation procedure, (b) energy switching procedure, (c) reaction field method, and (d) Ewald summation technique. Our analysis shows that the switching procedure with the short-range switching function introduces artifacts into the simulations. These artifacts are responsible for the faster decay and oscillations in the velocity autocorrelation function of the ions and therefore for the lower value of the diffusion coefficients. (C) 1995 American Institute of Physics.
\end{abstract}

\section{INTRODUCTION}

We are witnessing now a rapid development of computational hardware that will allow us to perform molecular dynamics computer simulations on systems with large number of particles and over long simulation times. To utilize the potential of the hardware, we have to develop reliable software and therefore resolve many challenging problems and answer many questions, old and new. Among the questions that need to be answered we find, for example, the question of how the treatment of long-ranged Coulomb forces influences the structure and dynamics of the system when we simulate an aqueous solution in the bulk. Many recent computer simulations clearly demonstrated that the values of the structural and thermodynamic properties of aqueous solutions strongly depend on the treatment of the long-range electrostatic forces. ${ }^{1-6}$

The usually adopted methods to treat long-ranged forces are to use some kind of cutoff procedure or the Ewald summation technique. In addition to these two methods, new ones, based on multipole expansions are now developed, ${ }^{7}$ but we are not going to address them in this work. The cutoff of the electrostatic interactions can be achieved by (i) applying a simple truncation procedure, (ii) multiplying the electrostatic potential or force by some kind of a switching function or a shifting function (when the width of a switching function is equal to zero, the switching procedure is equivalent to the truncation procedure), (iii) using the reaction field method. All of these cutoff procedures and the Ewald summation method introduce approximations into the treatment of the real systems and have their advantages and disadvantages. The use of switching or shifting functions modifies Coulomb interaction and forces it to zero after a certain distance. ${ }^{8}$ In the reaction field method the particles beyond the sphere with the certain cutoff radius $R_{c}$ are represented by a dielectric continuum. ${ }^{9}$ The advantage of the cutoff schemes is that they reduce computational demands on the system. In the Ewald summation method the periodic boundary conditions are fully utilized and the lattice sum is calculated. ${ }^{10}$ Sometimes this technique may produce artificial results due to the enhancement of the periodicity, while the real system is not periodic.

In many simulations the results depend on the way the electrostatic forces are treated. An interesting example, which illustrates dramatic influence of such a treatment on the structural and thermodynamic properties of aqueous solutions, is the calculation of the potential of mean force between two chloride anions in water. Earlier computer simulations predicted the $\mathrm{Cl}^{-}-\mathrm{Cl}^{-}$pairing in aqueous solutions. ${ }^{11}$ Later studies showed that the pairing disappeared when the Ewald summation procedure was used. ${ }^{3}$ A similar effect was observed for the ferrous-ferric system. ${ }^{12}$

While most of the previous work was devoted to the study of how the treatment of long-range forces influences structural and thermodynamics properties, much less is known of how these treatments influence the dynamical properties of the system. If one starts the study of thermodynamic properties of aqueous solutions with the potential of mean force, the proper place to start the study of the dynamical properties of aqueous solutions is to investigate the influence of long-range force treatment on the behavior of the ionic mobility, particularly the limiting ionic mobility. This was done recently by Rossky and collaborators ${ }^{13}$ who showed that for the bromide ion the velocity autocorrelation function and the value of the diffusion coefficient of this ion in water strongly depend on the treatment of long-range forces. They also found that the velocity autocorrelation function of bromide looked similar to the one of the Brownian particle when the Ewald summation technique was used. When the switching procedure was applied to the system the velocity autocorrelation function of the ion had an oscillatory character. Such oscillations in the velocity autocorrelation function were also observed previously for chloride ion in the simulations where the switching of the potential was used. ${ }^{14,15}$ In addition, the value of the diffusion coefficient obtained in Ref. 14 was around half the value measured in the experiment. The above-mentioned studies ${ }^{13-15}$ prompted us to ask the following questions: (a) how does the value of the chloride diffusion coefficient change when the Ewald summation is used to treat the long-ranged electrostatic force? (b) how does the qualitative character of the chloride 
velocity autocorrelation function change with the change in the long-range force treatment? While in the present work we will attempt to answer the above-asked questions related to chloride, the main goal of the present study is to understand how the treatment of the long-range forces influences the limiting ionic mobility of the anions.

\section{TREATMENT OF THE LONG-RANGE FORCES}

To treat the long-range forces we use four procedures in this work. They are: (i) the simple truncation procedure, (ii) a switching function procedure, (iii) the reaction field method, and finally (iv) the Ewald sum technique. The truncation procedure is the simplest one: in it we consider two molecules as interacting if the distance between their centers of mass is less than a cutoff radius $R_{c}$. If the distance is larger than $R_{c}$ the interaction is neglected. That means that we introduce an artificial discontinuity in our potential using this procedure. In the simulations with the switching procedure the discontinuity in the potential is avoided through the multiplication of the interaction with the switching function that starts reducing the potential at some distance $R_{L}$ and reduces it continuously to zero at some cutoff distance $R_{c}$. Therefore, the interaction between two particles $V_{1,2}$ is written as

$$
V_{1,2}=\sum_{i, j} V_{\mathrm{el}}\left(r_{i, j}\right) S(R),
$$

where the sum runs over all sites $i$ on particle 1 and all sites $j$ on particle $2 . R$ is the distance between two reference points on particle 1 and particle 2 . In our simulations we used the following switching function: ${ }^{16}$

$$
S(R)=\left\{\begin{array}{l}
1 \quad \text { if } R \leqslant R_{L} \\
1-\left(R-R_{L}\right)^{2}\left(3 R_{c}-R_{L}-2 R\right) /\left(R_{c}-R_{L}\right)^{3} . \\
0 \quad \text { if } R \geqslant R_{c}
\end{array}\right.
$$

Other forms of the switching function exist, but we use this particular one to be able to compare our present results with the previous simulations where the ionic mobility of the chloride ion was investigated. ${ }^{14,15}$ In our present simulations $R$ is the distance between oxygen atoms of water molecules when the interaction between two water molecules is considered. It is the distance between the oxygen of water and the ion, when the interaction between the ion and a water molecule is considered.

As was recently shown by Steinbach and Brooks ${ }^{17}$ (and as we shall see below) the switching procedure may result in the creation of large sudden changes in the force. To avoid these changes, the shifting procedure is used to turn off the potential continuously over long distance until it becomes zero at $R_{c}$. For example, some of the shifting functions used in the simulations of water and aqueous solutions have the general form ${ }^{18}$

$$
S(R)=\left[1-2\left(R / R_{c}\right)^{n}+\left(R / R_{c}\right)^{2 n}\right] \theta\left(R_{c}-R\right) .
$$

In Eq. (3) $\theta$ is the Heaviside step function and $n$ is some integer. Usually this shifting function is applied to every site-site Coulomb interaction, thus modifying it over the entire range of distances. For $n=1$ and $n=2$ the resulting modified electrostatic interactions were called MEI4 and MEI5, respectively, by Brooks et al. ${ }^{8}$ The shifting functions in these cases were called F1 and F2 by Prevost et al. ${ }^{18}$ Steinbach and Brooks ${ }^{17}$ recently introduced the force shift functions, that monotonically turn off the force at the distance $R_{c}$. With the application of such a function the force acting on site $i$ (with charge $q_{i}$ ) due to its Coulomb interaction with site $j$ (charge $q_{j}$ ) at a distance $R$, has the following form:

$$
\mathbf{F}(\mathbf{R})=\frac{q_{i} q_{j}}{R^{2}}\left[1-\left(R / R_{c}\right)^{\beta+1}\right] \frac{\mathbf{R}}{R} \theta\left(R_{c}-R\right),
$$

where $\beta$ is some integer.

In the reaction field method that we use in this work we check the distance between centers of mass of two molecules. If it is smaller than $R_{c}$ we assume that molecules interact and the force of interaction between sites $i$ and $j$ is given by the following expression: ${ }^{3}$

$$
\mathbf{F}\left(r_{i j}\right)=\frac{q_{i} q_{j}}{r_{i j}^{2}}\left[1-\left(r_{i j} / R_{c}\right)^{3}\right] \frac{\mathbf{r}_{i j}}{r_{i j}} \theta\left(R_{c}-R\right) .
$$

Equation (5) shows that the use of a reaction field method is equivalent to an application of a force shifting function of Eq. (4) with $\beta=2$.

Finally in the Ewald summation method we take full advantage of the periodic boundary conditions by periodically repeating our simulation box. To achieve convergence of the Coulomb sum we build up the periodic system in roughly spherical layers. Outside the sphere we have to specify the nature of the dielectric continuum surrounding the sphere, i.e., specify the dielectric constant $\epsilon_{r}$ of the continuum. There is a rather simple connection between the energy of the very large sphere of boxes surrounded by an ideal conductor $V\left(\epsilon_{r}=\infty\right)$ and the energy of the system embedded into vacuum $V\left(\epsilon_{r}=1\right)$. The connection is given by the following expression: ${ }^{19}$

$$
V\left(\epsilon_{r}=\infty\right)=V\left(\epsilon_{r}=1\right)-\frac{2 \pi}{3 \nu}\left|\sum_{i} q_{i} r_{i}\right|^{2},
$$

where $\nu$ is the volume of the unit cell. When the large sphere is in the vacuum, it has a dipolar layer on its surface. The last term in Eq. (5) is responsible for removing this layer, when the sphere is embedded in perfect conductor (tin foil boundaries). We run our Ewald simulations with these tin foil boundary conditions.

\section{MOLECULAR DYNAMICS SIMULATIONS}

In the present study, we have selected three systems involving fluoride, or chloride, or bromide ion solvated in 500 water molecules as well as a system involving one bromide ion solvated in 215 water molecules. Such a selection of different systems allows us to observe how the properties change in a molecular dynamics (MD) simulation due to the variation of the cutoff distance and the size of the ion. In the simulations with the fluoride and bromide ions the SPC water model $^{20}$ was chosen, while for the chloride ion, the TIP4P water model ${ }^{21}$ was used. The choice of different water 
TABLE I. Potential parameters used in the present study.

\begin{tabular}{lccc}
\hline \hline Water model & $A_{w-w} \mathrm{kcal} / \mathrm{mol} \AA^{12}$ & $C_{w-w} \mathrm{kcal} / \mathrm{mol} \AA^{6}$ & $q_{H}$ \\
\hline SPC & 629400.0 & 625.5 & 0.41 \\
TIP 4P & 600000.0 & 610.0 & 0.52 \\
Ion & $A_{w-I} \mathrm{kcal} / \mathrm{mol} \AA^{12}$ & $C_{w-I} \mathrm{kcal} / \mathrm{mol} \AA^{6}$ & $q_{I}$ \\
\hline Fluoride & 343100.0 & 426.2 & -1.0 \\
Chloride & 3950000.0 & 1461.2 & -1.0 \\
Bromide & 9118300.0 & 1911.8 & -1.0 \\
\hline \hline
\end{tabular}

models was primarily due to the fact that we can compare our current results with some of the previously obtained results from solvation studies of the above-mentioned ions under various simulation schemes. ${ }^{13-15}$ Also, we stress here that it is not our intention in the present study to emphasize the model performance relative to the reality, but to critically evaluate methods that are involved in the treatment of longrange interactions. All three ions were represented by a point charge having a Lennard-Jones (LJ) center on it. The Lennard-Jones parameters of the fluoride-SPC water ${ }^{22}$ and the chloride-TIP4P water ${ }^{23}$ interactions were the ones reported previously in the literature. The LJ parameters of the bromide-SPC water interaction were obtained after combining the parameters reported previously ${ }^{24}$ for bromide with the SPC water parameters. In all these cases we can write the interaction potential between the ion and a water molecule as

$$
V_{\mathrm{wI}}=\frac{A_{\mathrm{wI}}}{R_{\mathrm{oI}}^{12}}-\frac{C_{\mathrm{wI}}}{R_{\mathrm{oI}}^{6}}+\sum_{j} \frac{q_{I} q_{j}}{r_{I j}}
$$

and the interaction potential between two water molecules as

$$
V_{\mathrm{ww}}=\frac{A_{\mathrm{ww}}}{R_{\mathrm{oo}}^{12}}-\frac{C_{\mathrm{ww}}}{R_{\mathrm{oo}}^{6}}+\sum_{i j} \frac{q_{i} q_{j}}{r_{i j}} .
$$

In Eqs. (7) and (8) $R_{\mathrm{oI}}$ is the distance between the oxygen of the water molecule and the ion center. $R_{\mathrm{oo}}$ is the distance between oxygens of two water molecules. All the potential parameters along with the charges on the ions used in the current study are presented in Table I.

All our simulations were carried out in the NVT ensemble and the density was always selected to be $1 \mathrm{~g} / \mathrm{cc}$ at temperature $300 \mathrm{~K}$. For simulations with any one of the above-mentioned ions solvated in 500 water molecules, the required box length was $24.66 \AA$, whereas in the simulations with 215 water molecules the value was $18.62 \AA$.

Numerical evaluations of the trajectories were done using the leap-frog algorithm and the internal geometries of water molecules were constrained through the Shake procedure. To achieve the desired temperature, all the systems studied were coupled to a thermal bath ${ }^{25}$ with a coupling constant of $0.1 \mathrm{ps}$. All the trajectory calculations were performed with a time step of $2 \mathrm{fs}$. In all simulations the equilibration period of $40 \mathrm{ps}$ was followed by a period of $120 \mathrm{ps}$ for the data collection.

For each ion solvated in 500 water molecules, we have performed our molecular dynamics simulations using (1) the simple truncation procedure, (2) the reaction field method,
(3) a switching of the potential (which alters the force in the switching region), and (4) the Ewald summation procedure to treat long-range interactions. In the case of 216 particles in the primary box, we have selected to study only the case of bromide, since as it turned out in this case the four different methods produced the largest deviations in the large size system. This system with 216 particles was also studied using the same four methods to treat long-range forces as the ones mentioned above for the systems with 501 particles. In the Ewald scheme, the convergence parameter $\alpha$ had the value of $0.372 \AA^{-1}$ in order to confine the real space part of the sum to the primary simulation box. In the evaluation of the reciprocal space part, the maximum number of the components of the $k$ vectors had the value of 7 and $k^{2}<51$. Reaction field simulations were carried out with $\epsilon_{\mathrm{rf}}=\infty$ and the cutoff distances $R_{c}$ were 12 and $9.2 \AA$ in the simulations involving 500 and 215 water molecules, respectively. The same cutoff values were used in the simulations with the switching as well as the ones with the truncation. When the switching method was used, $R_{L}$ of Eq. (2) had a value of $0.95 R_{c}$ yielding a switching region of $0.6 \AA$ for the cutoff radius of $12 \AA$.

\section{RESULTS AND DISCUSSION}

The main goal of this work is to determine how the treatment of long-range forces influences the dynamics of ions in aqueous solutions, more specifically, the limiting ionic mobility. With this in mind, we have calculated the velocity autocorrelation functions of the ions. The results of the calculations are presented in Figs. 1(a), 1(b), and 1(c) where the velocity autocorrelation functions for fluoride, chloride, and bromide ions are shown. In each case, the correlation function was calculated from MD simulations with 500 water molecules, and using four different ways to treat long-range forces. The results from the present simulations for chloride, using the switching function, and for bromide, using both switching function and the Ewald sum method, are in agreement with the previously published results. ${ }^{13-15} \mathrm{~A}$ general feature common to Figs. 1(a)-1(c) is that the velocity autocorrelation function obtained from the simulation with the switching is displaying more oscillations compared to the correlation functions obtained from the MD with the other three methods. The difference is weak for fluoride, stronger for chloride, and very pronounced for the bromide ion. Also in cases of chloride and bromide the initial decay of the velocity autocorrelation function is enhanced when the switching function method is used in the simulations. The correlation functions obtained from the simulations with the Ewald method and with the reaction field are, within the statistical accuracy, practically the same. Surprisingly, the correlation functions from simulations with truncation look very similar to the correlation functions obtained from the simulations with the Ewald sum and with the reaction field.

To illustrate the influence of the cutoff value $R_{c}$ on the dynamic properties of the system, we display in Fig. 2 the velocity autocorrelation functions for bromide ion obtained from the simulations with 215 water molecules. From Figs. 2 and 1 (c) we observe that the largest change in the character 

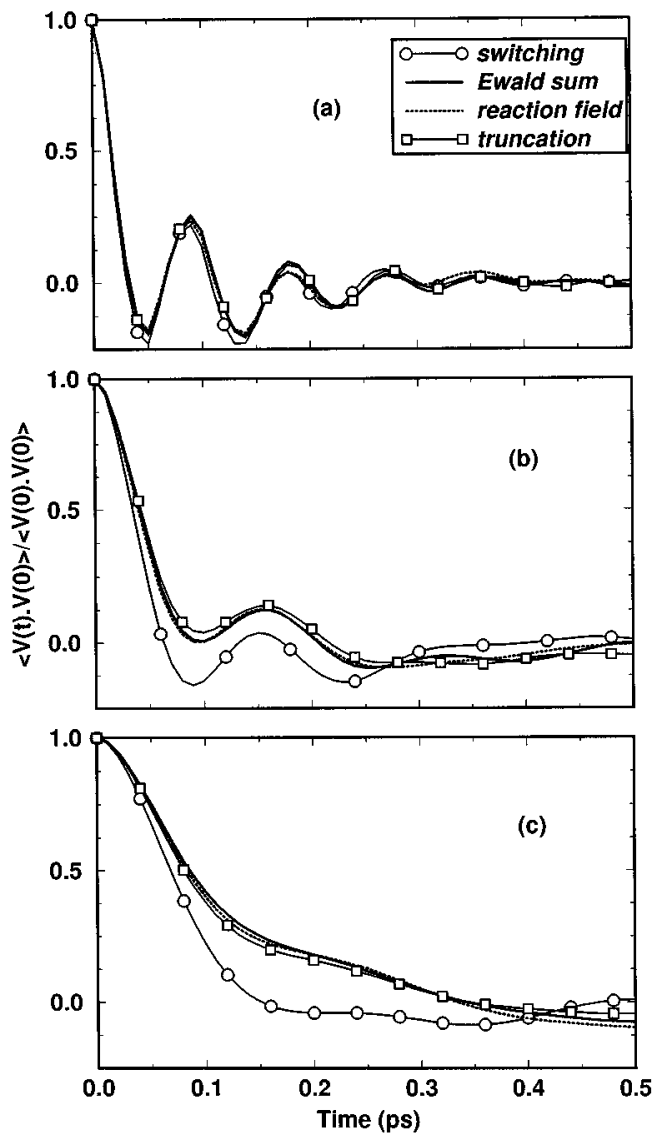

FIG. 1. Velocity autocorrelation functions of (a) fluoride, (b) chloride, and (c) bromide ions from the simulations with 500 water molecules.

of the correlation function occurs in the simulations where the switching function is used.

The enhanced oscillations and the fast initial decay in the velocity autocorrelation function obtained from the simulations with the switching functions are expected to result in a lower value of the diffusion constant, compared to the values of this constant obtained from the simulations where the other three methods were used. Indeed, when we calculated the values of the diffusion coefficients for the ions using the mean square displacement formula, that is what we observed. The mean square displacement curves, calculated for the whole 120 ps of the simulations are displayed in Fig. 3 for the chloride ion. Similar curves are obtained for the other ions. The values of the diffusion coefficients obtained from the fit of mean square displacement curves to straight lines in the interval of $1-3$ ps are presented in Table II(a). The change in the value of the diffusion coefficient cannot be attributed to the change in the coordination number of the ion, when different methods of long-range force treatments were used. The latter ones nearly do not change, as Table II(b) shows.

To see the effect of the ion size on the character of the ionic motion in water, we display the velocity autocorrelation functions for the fluoride, chloride, and bromide ions in Fig. 4. The correlation functions in Fig. 4 are obtained from the simulations with the Ewald summation. While the correlation

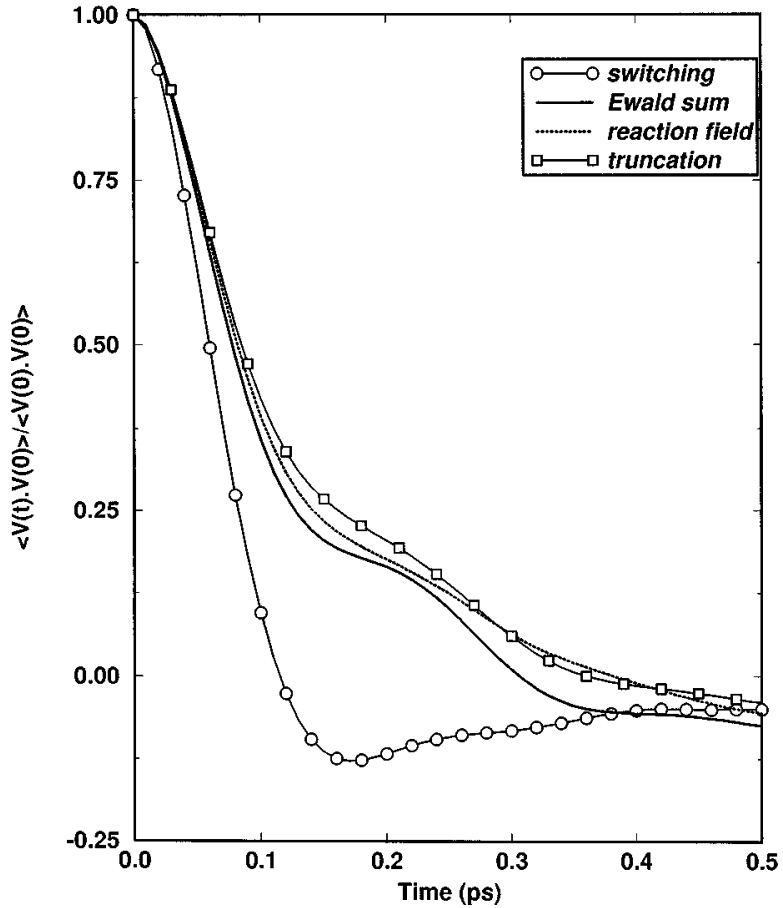

FIG. 2. Velocity autocorrelation functions of the bromide ion from the simulations with 215 water molecules.

function for fluoride displays strong oscillations, the oscillations are reduced for chloride and completely disappear from bromide. This different character of the ionic motion is rather simple to explain, if we neglect the effect of the ionic mass

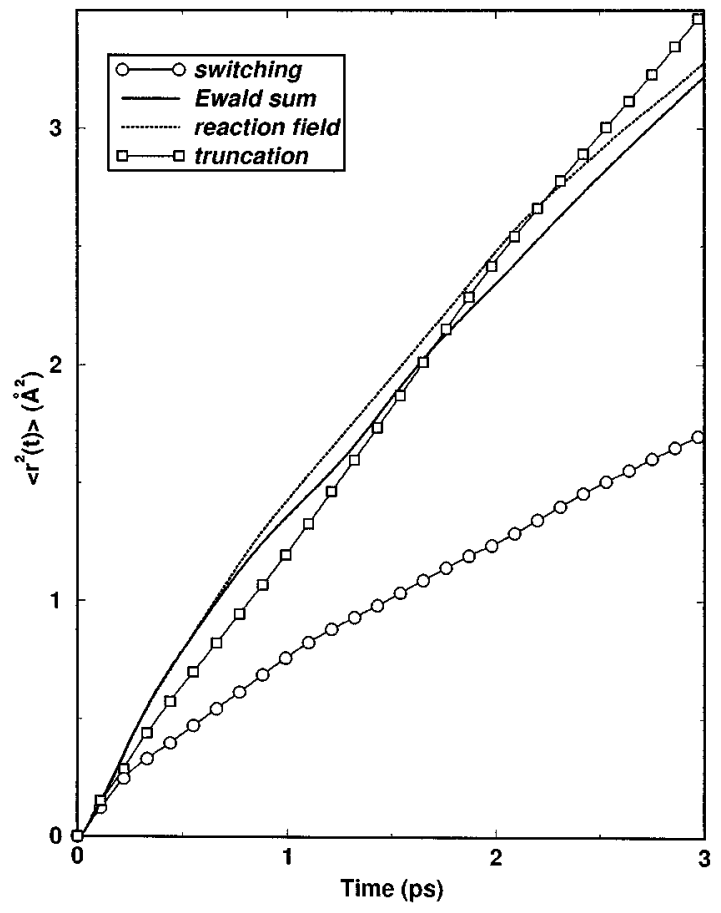

FIG. 3. Mean square displacements of the chloride ion from the simulations with 500 water molecules. 
TABLE II. (a) Diffusion coefficients (in $10^{-5} \mathrm{~cm}^{2} / \mathrm{s}$ ). (b) Coordination numbers.

\begin{tabular}{lccccc}
\hline \hline \multicolumn{1}{c}{ Ion } & Switching & $\begin{array}{c}\text { Reaction } \\
\text { field }\end{array}$ & $\begin{array}{c}\text { Ewald } \\
\text { sum }\end{array}$ & Truncation & Experiment $^{\mathrm{a}}$ \\
\hline (a) & & & & & \\
Fluoride & 1.56 & 2.06 & 1.72 & 1.80 & 1.46 \\
Chloride & 0.78 & 1.57 & 1.57 & 1.91 & 2.03 \\
Bromide & 1.37 & 2.43 & 2.34 & 2.50 & 2.08 \\
(b) & & & & & \\
Fluoride & 6.10 & 6.16 & 6.20 & 6.12 & \\
Chloride & 7.28 & 7.42 & 7.34 & 7.36 & \\
Bromide & 7.87 & 7.68 & 7.60 & 7.76 & \\
\hline \hline
\end{tabular}

${ }^{a}$ Reference 27.

on its motion. Due to the small size of the ion the electric field emanating from fluoride is strong. This keeps the water molecules around the ion for a long residence time, thus creating a cage around the ion. The ion therefore rattles in this cage, and the signature of this motion is observed as the oscillations in the velocity autocorrelation function. The cage effect is weaker for chloride and disappears for bromide. Figure 4 also shows that we cannot describe the motion of fluoride and chloride ions as Brownian, but the trend is clearly visible.

Since we know now that the simulations with the switching function result in an enhancement of the oscillatory motion, the main question is: why does this enhancement happen? A clue to the answer is obtained from Figs. 5(a), 5(b), and 6. In Figs. 5(a) and 5(b) we show the plots for the

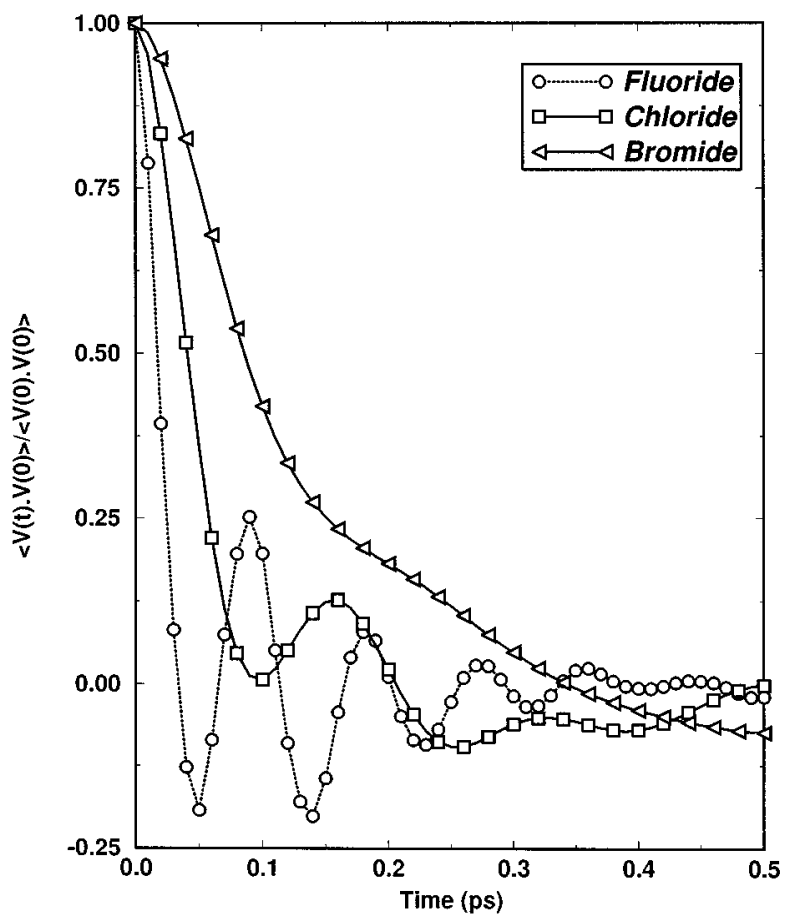

FIG. 4. Velocity autocorrelation functions of fluoride, chloride, and bromide ions from the simulations with 500 water molecules using the Ewald summation technique.

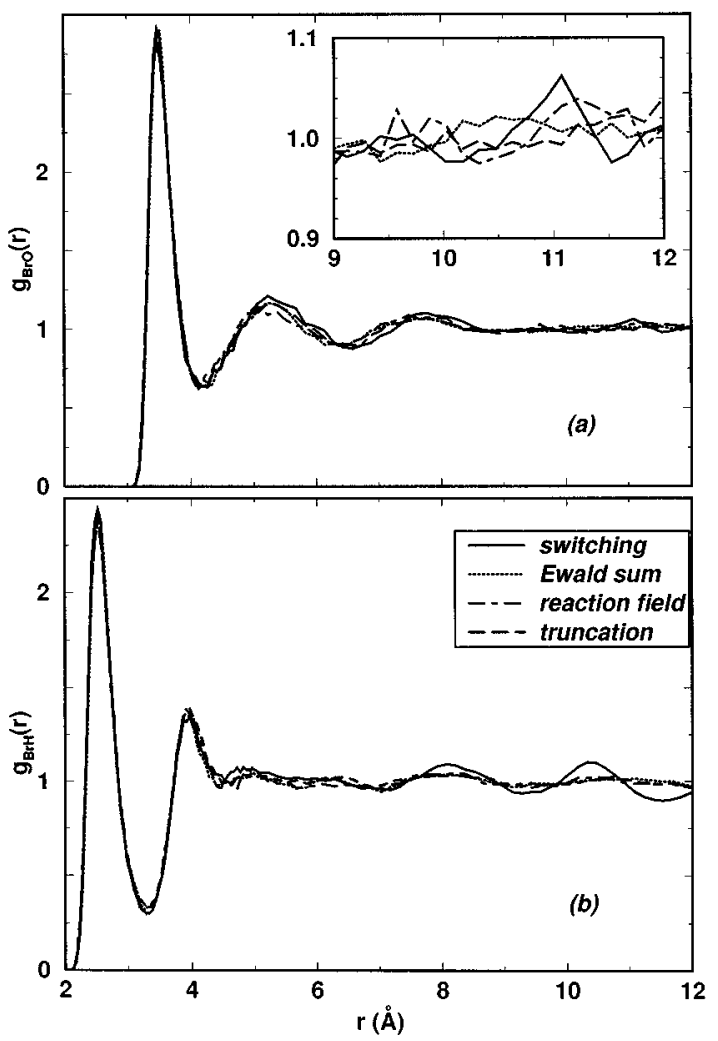

FIG. 5. (a) The bromide-oxygen radial distribution functions from simulations with 500 water molecules. The insert is the enlargement of the last $3 \AA$ segment. (b) The hydrogen-Bronide radial distribution functions obtained from the same set of simulations.

bromide-water oxygen [Fig. 5(a)] and the bromide-water hydrogen [Fig. 5(b)] radial distribution functions. (In what follows we show the distribution functions for bromide only, since we get the same qualitative information from the consideration of the other ion-water distribution functions.) As we can see from Fig. 5(a) the four methods produce statistically similar ion-water oxygen distribution functions in general, but when we use the potential switching method in the simulation, a spurious peak at around $11 \AA(0.4 \AA$ away from $R_{L}$ ) can be observed. Correspondingly, we observe the enhancement of hydrogen density at around $10.3 \AA$ in Fig. 5(b). In Fig. 6 we display the average cosine of the angle between the water dipole and the vector connecting the water oxygen with the ion center. As we can see from Fig. 6 a spurious peak is also observed for this distribution at the distance just before the switching function is turned on. Together, Figs. 5 and 6 indicate that the effect of the introduction of a switching function into the ion-water interaction potential is to create a buildup of water just before the distance $R_{L}$ from the ion, where the switching of the potential begins. Also, at this distance the water molecules in the simulations with the switching function display a preferential orientation of their dipoles towards the ion, which is easily inferred from the buildup of hydrogen and directly from Fig. 6. Thus we see, that when we use a switching function in our simulations, we create a shell of water around the ion close to the distance $R_{L}$. This shell outlines a large sphere of water around the 


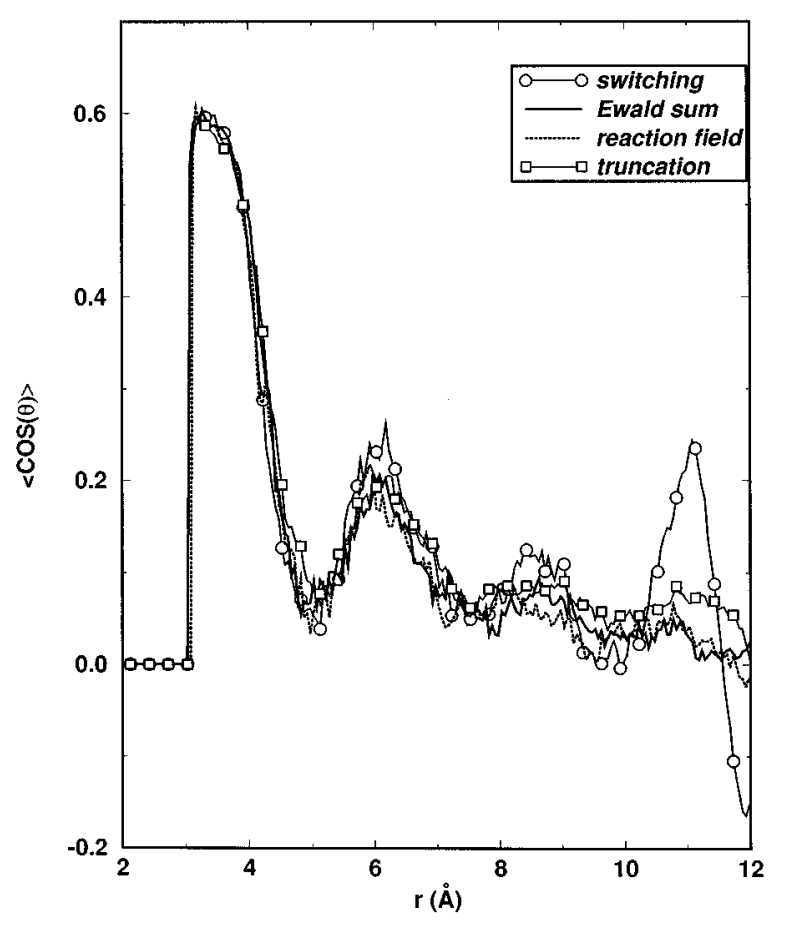

FIG. 6. Distributions of the cosine of the angle between the line joining the bromide ion and the water oxygen and the dipole vector as a function of distance from the ion.

ion, and the ion is performing a rattlelike motion in this large sphere, which is why we observe enhancement of the oscillations in the velocity autocorrelation function.

Why then do we observe the enhancement of the oxygen and particularly hydrogen density in the region where the action of the switching function starts? The answer can be obtained from the consideration of Fig. 7. Figure 7 displays how the truncation, reaction field, and switching procedures modify the Coulomb interaction between two similar charges. As we can see from Fig. 7, the reaction field modifies the interaction in a smooth manner and turns it off at $R=R_{c}$; the truncation procedure turns off the interaction abruptly at $R=R_{c}$. As we observe from Fig. 7, a large force is artificially created in the region of the switch, which is totally spurious. This large force can be either attractive or repulsive (depending on what atom it acts), but in any case it leads to a depletion of water in the switching region, which is responsible for the artifacts of the simulation.

Why then is the switching function method often used in the simulations? It seems that it was introduced into MD to avoid the warm up of the system, due to the energy discontinuity when the simple truncation method is employed alone. Although the switching function helps in stabilizing the value of the system's temperature, it can create more serious problems. Our results show that for the study of limiting ionic mobility the usage of the Ewald summation technique and of the reaction field technique (which is equivalent to the usage of the shifting of the force technique, proposed recently by Steinbach and Brooks) ${ }^{17}$ produced basically the same results. Previous simulation by Prevost et al. ${ }^{18}$ also demonstrated that the application of the shifting function [the

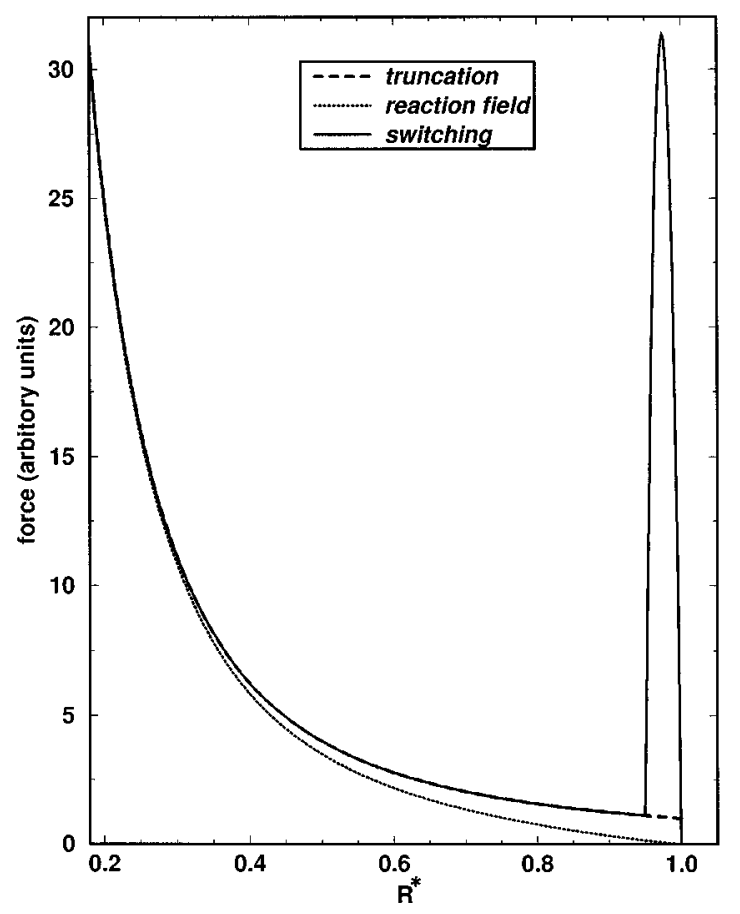

FIG. 7. The force between two charges as a function of $R^{*}\left(=R / R_{c}\right.$ under different cutoff schemes. The switching of the force begins at $0.95 R_{c}$.

one described in Eq. (3) with $n=1]$ to a Coulomb interaction potential produced the same results as the Ewald summation for pure water. The simple truncation method when applied to simulations with just one ion produced results which are very similar to the results from the simulations where Ewald and reaction field methods were used. However we do not recommend use of this simple truncation technique in simulations with more than one ion, since this cutoff scheme produces artificial correlations between ions. ${ }^{26}$

Our present study is in no way a comprehensive study of how the treatment of long-range forces influences the structure, thermodynamics, and dynamics of aqueous solutions. But on the basis of it we would recommend use of the Ewald summation or the reaction field techniques for the study of aqueous ionic solutions in computer simulations. At least these two methods produce consistent results and can be given a simple physical interpretation.

With respect to the questions asked in Sec. I and related to the change in the behavior of the velocity autocorrelation function and the value of the diffusion coefficient of the chloride ion, we observed that: (a) the velocity autocorrelation function of chloride is still oscillatory, even when Ewald summation or reaction field techniques are used to treat longrange interactions, (b) the value of the diffusion coefficient for chloride calculated using the Ewald summation technique is very close to the value obtained from the simulation using the reaction field method. Although these values are still below the experimental one by about $20 \%$, considering the uncertainty in the calculated value (estimated to be around $10 \%-15 \%$ ) the agreement between the calculation and experiment can be now considered as reasonable. 


\section{ACKNOWLEDGMENTS}

This work was supported by grants from the Office of Naval Research. Part of the simulations were performed on the Cray YMP at the North Carolina Supercomputer Center. We are grateful to Professor P. Rossky and Dr. B. Brooks for making preprints of Refs. 13 and 17 available to us prior to their publication.

${ }^{1}$ R. J. Loncharich and B. R. Brooks, Proteins: Structure, Function Genet. 6, 32 (1989).

${ }^{2}$ P. E. Smith and B. M. Pettitt, J. Chem. Phys. 95, 8430 (1991).

${ }^{3}$ G. Hummer, D. K. Soumpasis, and M. Neumann, Mol. Phys. 77, 769 (1992); 81, 1155 (1993)

${ }^{4}$ H. Alper and R. Levy, J. Chem. Phys. 91, 1242 (1989).

${ }^{5}$ D. M. York, T. A. Darden, and L. G. Pedersen, J. Chem. Phys. 99, 8345 (1993).

${ }^{6}$ H. Schreiber and O. Steinhauser, Chem. Phys. 168, 75 (1992); J. Mol. Biol. 228, 909 (1992); Biochemistry 31, 5856 (1992).

${ }^{7}$ L. Greengard and V. Rokhlin, J. Comp. Phys. 73, 325 (1987).

${ }^{8}$ C. L. Brooks III, B. M. Pettitt, and M. Karplus, J. Chem. Phys. 83, 5897 (1985).

${ }^{9}$ J. A. Barker and R. O. Watts, Mol. Phys. 26, 789 (1973).

${ }^{10}$ S. W. de Leeuw, J. W. Perram, and E. R. Smith, Proc. R. Soc. London, Ser. A 373, 27 (1980).
${ }^{11}$ L. X. Dang and B. M. Pettitt, J. Am. Chem. Soc. 109, 5531 (1987); J. Phys. Chem. 94, 4303 (1990).

${ }^{12}$ J. S. Bader and D. Chandler, J. Phys. Chem. 96, 6423 (1992).

${ }^{13}$ G. S. Del Buono, T. S. Cohen, and P. J. Rossky, J. Mol. Liq. 60, 221 (1994)

${ }^{14}$ M. R. Reddy and M. Berkowitz, J. Chem. Phys. 88, 7104 (1988).

${ }^{15}$ M. Berkowitz and W. Wan, J. Chem. Phys. 86, 376 (1987).

${ }^{16}$ O. Steinhauser, Mol. Phys. 45, 335 (1982).

${ }^{17}$ P. J. Steinbach and B. R. Brooks, J. Comp. Chem. 15, 667 (1994).

${ }^{18}$ M. Prevost, D. van Belle, G. Lippens, and S. Wodak, Mol. Phys. 71, 587 (1990).

${ }^{19}$ M. P. Allen and D. J. Tildesley, Computer Simulation of Liquids (Oxford University Press, Oxford, 1987).

${ }^{20}$ H. J. C. Berendsen, J. P. M. Postma, W. F. van Gunsteren, and J. Hermans, in Intramolecular Forces, edited by B. Pullman (Reidel, Dordrecht, 1981).

${ }^{21}$ W. L. Jorgensen, J. W. Madura, R. W. Impey, and M. L. Klein, J. Chem. Phys. 79, 926 (1983).

${ }^{22}$ T. P. Staatsma and H. J. C. Berendsen, J. Chem. Phys. 89, 5876 (1988).

${ }^{23}$ J. Chandrasekhar, D. C. Spellmeyer, and W. L. Jorgensen, J. Am. Chem. Soc. 106, 903 (1984).

${ }^{24}$ G. Palinkas, W. O. Riede, and K. Heinzinger, Z. Naturforsch. Teil A 32, 1137 (1977).

${ }^{25}$ H. J. C. Berendsen, J. P. M. Postma, W. F. van Gunsteren, A. DiNola, and J. R. Haak, J. Chem. Phys. 81, 3684 (1984).

${ }^{26}$ W. F. van Gunsteren (private communication).

${ }^{27}$ P. W. Atkins, Physical Chemistry (Freeman, New York, 1986). 